\title{
Safety of greenlight photoselective vaporisation of prostate in lower urinary tract symptoms due to benign prostatic hyperplasia in patients using anticoagulants due to cardiovascular comorbidities
}

\author{
Basri Cakiroglu ${ }^{1}$, Orhun Sinanoglu ${ }^{2}$, Akif Nuri Dogan ${ }^{3}$ \\ ${ }^{1}$ Hisar Intercontinental Hospital, Department of Urology, Umraniye, Istanbul, Turkey; \\ 2 Maltepe University Department of Urology Maltepe, Istanbul, Turkey; \\ ${ }^{3}$ Hisar Intercontinental Hospital, Department of Internal Medicine, Umraniye, Istanbul, Turkey.
}

\begin{abstract}
Summary Lasers have been used in the management of benign prostatic hyperplasia for the last two decades. To be comparable, they should reduce or avoid the immediate and long-term complications of transurethral resection of the prostate (TURP) or open prostatectomy (OP), especially bleeding and need for blood transfusion. Although Holmium laser treatment of the prostate was compared frequently in terms of cardiovascular safety with TURP or OP, photoselective vaporisation of the prostate (PVP) was not largely evaluated. In this article we analyzed the current literature to see if there is convincing data to support the observation of some authors that use of PVP is associated with increased safety in patients on anticoagulants with cardiovascular comorbidities. With this purpose a Medline search between January 2004 to March 2013 was performed using evidence obtained from randomised trials, well-designed controlled studies without randomisation, individual cohort studies, individual case control studies and case reports

Results: In the last 10 years, several case-control and cohort studies have demonstrated the efficacy of PVP as well as its safety in patients with cardiovascular comorbidities using anticoagulants. The results confirmed the overall lower perioperative and postoperative morbidity of PVP, whereas the efficacy was comparable to TURP in the short term, despite a higher reoperation rate.

Conclusion: Although it is still developing, PVP with KTP or LBO seems to be a promising alternative to both TURP and $O P$ in terms of cardiovascular safety and in patients using anticoagulants.
\end{abstract}

KEY WORDS: Anticoagulant therapy; Benign prostate hyperplasia; Cardiovascular comorbidities; Photoselective prostate vaporization.

Submitted 6 February 2015; Accepted 15 March 2015

\section{INTRODUCTION}

Transurethral resection of the prostate (TURP) is the gold standard of surgical treatment of BPH however, several complications such as incontinence, uretral stricture, impotence, TUR syndrome as well as cardiovascular adverse events due to bleeding may occur (1). Cardiovascular as well as cerebrovascular diseases increase with age and these patients require antithrombotic treatments, therefore alternative techniques have been developed for patients with blood coagulation disorders secondary to anticoagulants or antiplatelet intake. Currently, holmium laser enucleation of the prostate (HoLEP) and photoselective vaporization of the prostate (PVR) (with potassium titanyl phosphate [KTP]) are the most studied technical modalities suggesting that they may be valid alternatives to both open prostatectomy (OP) and transurethral resection of the prostate (TURP) (2). PVP by Green Laser is an ablative technique with simultaneous hemostasis (3). This technique enables rapid and effective vaporization of the obstructing prostate adenoma, with adequate cavitation and minimal morbidity. KTP laser emits visible green light and has 532 nm wavelenght which is absorbed by hemoglobin, but not by water. It vaporizes the tissue without loss of energy in liquid setting with consequent vaporisation. Although patients with normal hemostatic parameters with normal international normalized ratio (INR) do not have bleeding complications, the usage of this procedure in patients with anticoagulant therapy remains an important issue. The leading concerns for TURP have been intra-operative bleeding and the need for blood transfusion. The risk is higher in patients on anticoagulants and antiplatelet agents. The great majority of publications evaluating prostate surgery in conjunction with anticoagulants evaluated men who take either on aspirin, clopidogrel and coumadin (4).

In the present review, we have attempted to analyze the current literature to see if there is convincing data to support the observation of some authors that use of PVP is associated with lower cardiovascular events and increased safety in patients on anticoagulants.

A Medline search over the last 10 years January 2004 to March 2014 was performed using evidence obtained from randomised trials, well-designed controlled studies without randomisation, individual cohort studies, individual case control studies and case reports. 


\section{TECHNICAL ASPECT}

The green light photoselective vaporisation of the prostate (PVP) is generated bypassing a neodymium:yttrium aluminium garnet (Nd:YAG) laser with 1064-nm laser light through a frequency-doubling crystal, reducing the wavelength by half to $532 \mathrm{~nm}$. The older 80-W laser device uses the KTP (potassium titanyl phosphate) crystal, and the latest appliance uses the LBO (lithium borate) crystal with a $120-\mathrm{W}$ power setting. The high absorption of the laser, predominantly by hemoglobin rather than by water, offers enhanced hemostatic properties; therefore, vaporisation is achieved by concentrating heat in a small volume within a very short period of time. The use of saline as irrigating solution avoids the risk of TUR syndrome. These findings are of particular interest when using PVP for large prostates, as an alternative to OP or TURP. The inconvenience of PVP is the lack of specimen for pathologic assessment (5).

\section{THE SUPERIORITY OF PVP FOR PATIENTS ON ANTICOAGULANT DRUGS WITH CARDIOVASCULAR COMORBIDITY}

TURP is considered a gold standard in management of $\mathrm{BPH}$. For larger prostates open prostatectomy (OP) remains the best option. The search of alternatives for both modalities continues considering their disadvantages. One of the most important adverse events for TURP and OP has been intra-operative bleeding and the necessity for blood transfusion (6).

This risk is higher in patients on anticoagulants and antiplatelet agents. Additionally intravasation of hypotonic fluid and risk of TUR syndrome is high in large prostates causing TUR syndrome due to hyponatremia hypoosmolar cellular swelling with hypervolemia which is a burden for patients with high cardiovascular risks. One of the major advantages of PVP is its bloodless nature. In a series, PVP was performed successfully in 66 patients with high cardiac risk, presented with an American Society of Anesthesiology score of 3 or more (7). Within these 66 patients 29 patients were on oral anticoagulant therapy or had a severe bleeding disorder. No intra-operative or postoperative major complication occurred and no blood transfusion was required. Furthermore, 77\% patients did not require irrigation post-operatively. Mean catheterization time was $1.8 \pm 1.4$ days. Two patients required reoperation due to urinary retention. Many other studies suggest PVP as a treatment option in patients with benign prostate hyperplasia who are at high risk for clinically significant bleeding $[8,9]$. Sandhu and colleagues reported the results of the 80-W KTP laser in 24 men receiving various forms of anticoagulation (8). Of the 24 men, 8 were taking warfarin, 2 clopidogrel, and 14 aspirin. These patients displayed an increased prevalence of cardiovascular disease: 8 (33\%) had a history of myocardial infarction, 7 (29\%) of cerebrovascular disease and 7 (29\%) of peripheral vascular disease. After laser prostatectomy, no patient developed clinically significant hematuria postoperatively. There were no episodes of clot retention. No transfusions were required. Overall, all patients underwent PVP safely without any adverse thromboembolic or bleeding events. One patient had transient postoperative urinary retention requiring catheterization, two patients developed retrograde ejaculation, and two patients had urinary tract infections postoperatively (8). The safety of PVP series in high-risk anticoagulated patients was further studied in a larger series of 83 patients by Malloy and colleagues (9). Eighty-one of the patients had a defect in coagulation parameters at the time of surgery. Immediate postoperative electrolytes and hemoglobin showed no significant changes, with minimal intraoperative blood loss. No transfusions were required, and there were not any thromboembolic events. Five patients experienced clot retention in the immediate postoperative period. Overall, studies with PVP have shown that it is safe for patients with coagulopathies, platelet disorders, and those considered to be high cardiovascular or cardiopulmonary surgical risks. Other studies suggest that PVP combines both ablation and coagulation creating the opportunity for nearly bloodless surgery (10). For this convenience, the method could be used for treatment of larger prostates. It also provides safe and effective treatment in patients with high cardiovascular or pulmonary risks receiving anti-coagulant therapy and in patients with acute urinary retention $(4,11)$. The results of 80 -W KTP laser PVP has also been reported in a large series with 500 patients: no TUR syndrome occurred, and only two patients required blood transfusions. Despite oral anticoagulation in $45 \%$ of the patients, no severe intraoperative complications occurred. The results are presented as a success compared to TURP in patients with comorbidities. However the studies before 2008 were performed with PVP using 80-W-KTP with the low rate of vaporization; thus, in patients with a large prostate volume, long operative times were needed (12). A new KTP laser, the 120-W GreenLight HPS, was introduced to overcome this flaw. The new system emits the same green wavelength with a power of $120 \mathrm{~W}$, resulting in higher vaporization speed. The PVP with 120-W HPS provides the possibility of faster tissue vaporization while keeping the hemostatic advantages of its predecessor.

The International Green Light User group reported treatment results of PVP with 120-W HPS in 305 patients. They concluded that that the HPS can be used effectively and safely in patients receiving anticoagulant and patients with urinary retention or those with a larger prostate (4). In a recent study the outcomes of PVP in 45 patients taking oral anticoagulant therapy and in which withdrawal would have posed a considerable risk of thromboembolic events were reported. Furthermore, the study included one patient who had chronic myelogenous leukemia, which is a also severe bleeding disorder. PVP was performed safely in these patients on full anticoagulation, and no thromboembolic or bleeding events occurred (13). In a latest study, 63 patients under anticoagulant treatment undergoing 120-W GreenLight HPS prostatectomy had considerable symptomatic improvement without bleeding or thromboembolic event (14). In conclusion, KTP and LBO lasers seems to have low risk of bleeding complications in this patient population. Other commonly used laser technologies, such as the 2013 nm thulium [Revolix (Lisa Laser, Pleasanton, California, USA)] and the $980 \mathrm{~nm}$ lasers [Evolve (Biolitec, Inc, East Longmeadow, Massachusetts, USA)], have not been thoroughly studied in patients receiving to antico- 
agulation. The two series evaluating HoLEP (Holmium laser enuclation of prostate) in patients on anticoagulation in which warfarin and aspirin was continued during surgery reported a higher $8 \%$ abortion rate of the procedure and a $10 \%$ transfusion rate (15).

\section{Conclusion}

Patients undergoing prostate surgery are generally older with more comorbidities and frequently receive antithrombotic therapy for cardiovascular diseases. Physicians do not stop these medications through prostate surgery to avoid the risk of cardiovascular and cerebrovascular complications. From the limited retrospective, noncomparative studies available, PVP appear to provide superior hemostasis. Future investigation with prospective, randomized studies of patients on commonly used oral anticoagulant therapies comparing various lasers with ablative technical modalities is necessary to make definitive conclusions on the most effective technique with the fewest complications.

\section{REFERENCES}

1.Madersbacher S, Alivizatos G, Nordling J, et al. EAU 2004 guidelines on assessment, therapy and follow-up of men with lowerurinary tract symptoms suggestive of benign prostatic obstruction (BPH guidelines). Eur Urol. 2004; 46:547-554.

2. Reich $O$, Gratzke C, Stief CG. Techniques and long-term results of surgical procedures for BPH. Eur Urol. 2006; 49:970-8.

3. Malek RS, Nahen K. Photoselective vaporisation of the prostate: KTP laser therapy of obstructive benign prostatic hyperplasia. AUA Update Ser. 2004; 23:153-9.

4. Woo H, Reich O, Bachmann A, et al. Outcome of GreenLight HPS 120-W laser therapy in specific patient populations: Those in retention, on anticoagulants, and with large prostates (> $80 \mathrm{ml}$ ) Eur Urol Supp. 2008; 7:378-83.
5. Kuntz RM. Current role of lasers in the treatment of benign prostatic hyperplasia (BPH). Eur Urol. 2006; 49: 961-9.

6. Fitzpatrick JM. Minimally invasive and endoscopic management of benign prostatic hyperplasia. In: Wein AJ, Kavoussi LR, Partin AW, Novick AC, Peters CA, editors. Campbell Walsh Urology. 10th ed. Philadelphia: Elsevier Saunders; 2012. pp. 2655-94.

7. Reich O, Bachmann A, Siebels M, et al. High power ( $80 \mathrm{~W}$ ) potassium-titanyl-phosphate laser vaporization of the prostate in 66 high risk patients. J Urol. 2005; 173:158-60.

8. Sandhu JS, Ng CK, Gonzalez RR, Kaplan SA, Te AE. Photoselective laser vaporization prostatectomy in men receiving anticoagulants. J Endourol. 2005; 19:1196-8.

9. Yuan J, Wang H, Wu G, et al. High-power ( $80 \mathrm{~W}$ ) potassium titanyl phosphate laser prostatectomy in 128 high-risk patients. Postgrad Med J. 2008; 84:46-9.

10. Rajbabu K, Chandrasekara SK, Barber NJ, et al. Photoselective vaporization of the prostate with the potassium titanyl-phosphate laser in men with prostates of $>100 \mathrm{~mL}$. BJU Int. 2007; 100:593-8.

11. Yuan J, Wang $H$, Wu G, et al. High-power ( 80 W) potassium titanyl phosphate laser prostatectomy in 128 high-risk patients. Postgrad Med J. 2008; 84:46-9.

12. Ruszat R, Seitz M, Wyler SF, et al. Greenlight laser vaporization of the prostate: single-center experience and long-term results after 500 procedures. Eur Urol. 2008; 54:893-901.

13. Wei Tao, BoXin Xue, Yachen Zang, et al. The application of 120W high-performance system Green Light laser vaporization of the prostate in high-risk patientsLasers Med Sci. 2013; 28:1151-1157.

14. Cakiroglu B, Gözüküçük R, Sinanoglu O. Efficacy and safety of $120 \mathrm{w}$ greenlight photoselective vaporisation of prostate in patients receiving anticoagulant drugs.J Pak Med Assoc. 2013; 63:1464-7.

15. Wei Tao, BoXin Xue, Yachen Zang, et al. The application of 120W high-performance system GreenLight laser vaporization of the prostate in high-risk patients Lasers Med Sci. 2013; 28:1151-1157.

\section{Correspondence}

Basri Cakiroglu, MD (Corresponding Author)

drbasri@yahoo.com

Department of Urology - Hisar Intercontinental Hospital

Saray Mah. Site Yolu Caddesi No:7 - Umraniye, Istanbul, Turkey

Orhun Sinaniglu, MD

Department of Urology - Maltepe University Department of Urology

Maltepe, Istanbul, Turkey

Akif Nuri Dogan, MD

Department of Internal Medicine - Hisar Intercontinental Hospital

Umraniye, Istanbul, Turkey 